\title{
Lack of Association between Pre-Operative Insulin-Like Growth Factor-1 and the Risk of Post-Operative Delirium in Elderly Chinese Patients
}

\author{
Che-Sheng Chu', Chih-Kuang Liang ${ }^{2,3,4}$, Ming-Yueh Chou ${ }^{2,5}$, Yu-Te Lin², \\ Chien-Jen Hsu ${ }^{5}$, Chin-Liang $\mathrm{Chu}^{2,6} \bowtie$, and Po-Han $\mathrm{Chou}^{7,8} \bowtie$ \\ ${ }^{1}$ Department of Psychiatry, Puli Branch, Taichung Veterans General Hospital, Taichung, Taiwan \\ ${ }^{2}$ Center for Geriatrics and Gerontology, Kaohsiung Veterans General Hospital, Kaohsiung, Taiwan \\ ${ }^{3}$ Division of Neurology, Department of Medicine, Kaohsiung Veterans General Hospital, Kaohsiung, Taiwan \\ ${ }^{4}$ School of Medicine, National Yang-Ming University, Taipei, Taiwan \\ ${ }^{5}$ Department of Medical Education, Kaohsiung Veterans General Hospital, Kaohsiung, Taiwan \\ ${ }^{6}$ Department of Psychiatry, Kaohsiung Veterans General Hospital, Kaohsiung, Taiwan \\ ${ }^{7}$ Department of Psychiatry, Taichung Veterans General Hospital, Taichung, Taiwan \\ ${ }^{8}$ Department of Psychiatry, School of Medicine, National Yang-Ming University, Taipei, Taiwan
}

Objective Postoperative delirium (POD) is a highly prevalent complex neuropsychiatric syndrome in elderly patients. However, its pathophysiology is currently unknown. Early detection and prevention of POD is important; therefore, the aim of this study was to investigate the link between preoperative insulin growth factor 1 (IGF-1) levels in the serum and POD in the Chinese elderly patients.

Methods One hundred and three patients who were undergoing an orthopedic operation took part in the study. Preoperative serum IGF-1 levels were measured. POD was determined daily using the Confusion Assessment Method (CAM) and DSM-IV TR. Baseline serum IGF-1 levels were compared between patients who did and did not develop POD. Correlation coefficients were calculated to evaluate relationship between baseline characteristics and serum IGF-1 levels. The relationship between baseline biomarkers and delirium status was investigated using logistic regression analysis, adjusting for potential confounding variables.

Results Twenty-three patients developed POD. The POD group had lower MMSE scores and higher CCI scores and proportions of acute admission. Preoperative serum IGF-1 levels were correlated with MMSE scores and age (MMSE: $r=0.230$, $p<0.05$; age: $r=-0.419$, $\mathrm{p}<0.001$ ). Baseline serum IGF-1 levels did not differ between patients who did and did not develop POD, even after adjusting for potential confounding factors, MMSE score, and age.

Conclusion No association was found between preoperative IGF-1 levels and POD, suggesting that they are not direct biomarkers of the incidence of POD among the Chinese elderly population. Further research with larger sample sizes is warranted to clarify the relationship.

Psychiatry Investig 2016;13(3):327-332

Key Words IGF-1, Postoperative delirium, Elderly.

\section{INTRODUCTION}

Delirium, or acute confusional state, is a clinical neuropsychiatric syndrome characterized by acute and fluctuating impairments in attention and cognitive dysfunction. Dementia, sleep deprivation, and immobilization have been show to predict delirium. Postoperative delirium (POD) is delirium that happens to individuals who received a surgery. Depending on the age of patients and type of surgery, POD rates vary. For example, POD is estimated to occur in $35 \%$ and $28 \%$ to $60 \%$

\footnotetext{
Received: June 1, 2015 Revised: July 13, 2015 Accepted: August 23, 2015 Available online: January 11, 2016

$\triangle$ Correspondence: Po-Han Chou, MD

Department of Psychiatry, Taichung Veterans General Hospital, 1650, Taiwan Boulevard Sect. 4, Taichung, Taiwan 40705, R.O.C.

Tel: +886-4-23592525, Fax: +886-4-24619627, E-mail: phchou1980@gmail.com

$\triangle$ Correspondence: Chin-Liang Chu, MD

Department of Psychiatry, Kaohsiung Veterans General Hospital, No. 386, Ta-Chung 1st Road, Kaohsiung, 81362, Taiwan, R.O.C.

Tel: +886-7-3422121\#2067, Fax: +886-7-3468346, E-mail: mdjim0814@gmail.com

(a) This is an Open Access article distributed under the terms of the Creative Commons Attribution Non-Commercial License (http://creativecommons.org/licenses/by-nc/3.0) which permits unrestricted non-commercial use, distribution, and reproduction in any medium, provided the original work is properly cited.
} 
of patients after vascular operation and after operation for hip fracture, respectively. ${ }^{1,2}$ POD contributes to increased mortality rates, institutionalization, delayed functional recovery, and prolonged hospital stays, ${ }^{1,3}$ however, its pathophysiology is poorly understood and if often neglected by medical professionals. ${ }^{4,5}$

Various hypotheses for the pathogenesis of delirium have been proposed, including abnormalities in neurotransmission, dysregulation of the stress response, anti-inflammatory reactions. ${ }^{6}$ This complex pathophysiology makes diagnosis and disease prevention difficult; however due to its clinical importance, a number of studies have emerged investigating serum cytokines and their association with the development of delirium. In particular, biomarkers, such as insulin growth factor 1 (IGF-1), have been intensively investigated. ${ }^{7-13}$

One common putative pathophysiologcial mechanism linking IGF-1 to delirium was direct neuroprotection effects of IGF- $1 .{ }^{14}$ IGF-1 is a neuroprotective cytokine that plays a vital role in promoting development, regulating cell proliferation, differentiation, and synaptogenesis. ${ }^{15}$ IGF-1-knockout mice are $40-45 \%$ smaller than their wild-type littermates and IGF1 deficiency in humans causes growth abnormalities, including a smaller brain and mental retardation. ${ }^{16,17}$ In addition, lower serum IGF-1 levels are linked to poor cognitive performance, and early administration of IGF-1 after a neural injury, such as stroke, reduces secondary neuronal loss. ${ }^{18}$ These findings suggest a neuroprotective role of IGF-1 in the central nervous system. ${ }^{5}$

On the other hand, other studies proposed that the role of IGF-1 in delirium may be due to direct interaction with other cytokines, such as IFN-gamma, IL-6, TNF-alpha, and IL-1beta. ${ }^{14}$ For example, therapeutic use of IFN-gamma can cause delirium and IGF-1 can be regulated by IFN-gamma. ${ }^{19,20} \mathrm{~Pa}$ tients with low IGF-1 levels may lack an innate immune response against the response of cytotoxic cytokines due to physical or chemical insults. This would make them more vulnerable to the development of delirium. However, there are inconsistent findings in the literature, with some studies showing an association between lower serum IGF-1 levels and delirium, while others reveal no association. ${ }^{7-13}$

There are several possible reasons for this inconsistency. First, the majority of the studies analyzing correlations between IGF-1 markers and delirium used acutely admitted patients in medical wards. These results are likely to be confounded by underlying medical illnesses. ${ }^{7,8,10,12,13}$ Second, the studies had different experimental designs. Blood sampling and delirium occur on the same day in the majority of the studies; therefore, it is not possible to ascertain whether serum levels of IGF-1 are the cause of delirium or reflected a concurrent change due to acute illness. ${ }^{7,810}$ Third, previous studies have been conducted in Western countries; therefore, it is not known whether these findings can be generalized to other ethnic populations. ${ }^{7-13}$ In the present study, we sought to investigate the association between IGF-1 levels and the incidence of delirium in a homogeneous and well-defined population. Cytokine concentrations were measured preoperatively in elderly patients who had been admitted for orthopedic surgery and were not acutely ill.

\section{METHODS}

\section{Setting and subjects}

Between March 2012 and March 2013, we approached elderly patients aged 75 years or above, that were admitted to the orthopedic ward of a medical center in Southern Taiwan for acute or elective vertebral, knee, or hip surgery. Informed consent was obtained from all participants at the time of enrollment. Patients were excluded if 1 ) they had been prescribed cholinesterase inhibitors or NMDA receptor antagonists during the research period; or 2) they were delirious at the time of admission. The study protocol was approved by the institutional review board at Kaohsiung Veteran General Hospital.

\section{Clinical assessment}

Demographic and general clinical details, including age, sex, years of education, type of admission (outpatient or emergency department), and type of surgery (vertebral, hip, or knee) were recorded. Patients were assessed using the Barthel Index, ${ }^{21}$ Geriatric Depression Scale (GDS-15), ${ }^{22}$ CAGE questionnaire, ${ }^{23}$ Mini-Mental State Examination (MMSE), ${ }^{24}$ and Charlson Comorbidity Index (CCI). ${ }^{25}$ Further, they were assessed for polypharmacy ( $\geq 4$ prescriptions). Visual acuity and hearing ability were tested using the Snellen eye chart and Whisper test, respectively. ${ }^{26}$ The Confusion Assessment Method (CAM) criteria were used by a trained researcher to screen for possible postoperative delirium daily. ${ }^{27}$ Visual Analogue Scale (VAS) was conducted for the assessment of intensity of pain.

Delirium was diagnosed by a research psychiatrist according to the Diagnostic and Statistical Manual of Mental Disorders, 4th Edition, Text Revision (DSM-IV TR) criteria.

\section{Laboratory assessments}

Two tubes of blood ( $15 \mathrm{~mL}$ each) were drawn from the patients the morning before their surgery. One sample was used for white blood cell counts and hemoglobin measurements. The second sample was centrifuged to obtain serum and stored at $-80^{\circ} \mathrm{C}$ for future analysis. A sandwich ELISA kit (Biovendor, $\mathrm{EU})$, modified for optimal sensitivity, was used to quantify the serum concentration of IGF-1 according to the manufacturer's instructions. 
Table 1. Group comparisons of baseline characteristics and other covariables between subjects with POD and those without POD

\begin{tabular}{|c|c|c|c|c|}
\hline Variables & $\begin{array}{l}\text { All enrolled patients } \\
\qquad(\mathrm{N}=103)\end{array}$ & $\begin{array}{l}\text { Non-delirium patients } \\
\qquad(\mathrm{N}=80)\end{array}$ & $\begin{array}{l}\text { Delirium patients } \\
\qquad(\mathrm{N}=23)\end{array}$ & $\mathrm{p}$ \\
\hline \multicolumn{5}{|l|}{ Baseline } \\
\hline Age, years & $81.74 \pm 3.98$ & $81.91 \pm 3.63$ & $81.13 \pm 5.05$ & 0.494 \\
\hline Male gender, N (\%) & $76(64.4)$ & $58(72.5)$ & $18(78.3)$ & 0.789 \\
\hline Admission route, $\mathrm{N}(\%)$ & & & & $<0.05$ \\
\hline Emergency room & $15(12.7)$ & $8(10.0)$ & $7(30.4)$ & \\
\hline Outpatient clinic & $88(74.6)$ & $72(90.0)$ & $16(69.6)$ & \\
\hline Education, years & $6.15 \pm 4.76$ & $6.19 \pm 4.83$ & $6.00 \pm 4.59$ & 0.188 \\
\hline Current smoker, N (\%) & $7(5.9)$ & $4(5.0)$ & $3(13.0)$ & 0.184 \\
\hline Polypharmacy, N (\%)* & $50(42.4)$ & $36(45.0)$ & $14(60.9)$ & 0.238 \\
\hline Psychopharmacy, N (\%) & $12(10.2)$ & $8(10.0)$ & $4(17.4)$ & 0.459 \\
\hline Hearing impairment, N (\%) & $25(21.2)$ & $18(22.5)$ & $7(30.4)$ & 0.335 \\
\hline Vision & $79(66.9)$ & $61(76.3)$ & $18(78.3)$ & 0.159 \\
\hline Impairment, N (\%) & $46(39)$ & $37(46.3)$ & $9(39.1)$ & 0.637 \\
\hline BMI & $25.24 \pm 3.35$ & $25.82 \pm 3.09$ & $23.22 \pm 3.48$ & $<0.05$ \\
\hline Barthel Index ${ }^{\dagger}$ & $91.07 \pm 15.71$ & $92.44 \pm 14.32$ & $86.3 \pm 19.43$ & 0.408 \\
\hline $\mathrm{IADL}^{\ddagger}$ & $5.33 \pm 1.70$ & $5.54 \pm 1.58$ & $4.61 \pm 1.90$ & $<0.05$ \\
\hline MMSE & $23.09 \pm 5.21$ & $24.50 \pm 4.16$ & $18.17 \pm 5.60$ & $<0.001$ \\
\hline GDS-15§ & $0.96 \pm 2.72$ & $0.95 \pm 2.69$ & $1.0 \pm 2.88$ & 0.092 \\
\hline $\mathrm{CCI}$ & $1.05 \pm 1.25$ & $0.86 \pm 1.02$ & $1.7 \pm 1.71$ & $<0.05$ \\
\hline Hospitalization, days & $8.65 \pm 2.71$ & $8.62 \pm 2.87$ & $8.74(7-11)$ & 0.385 \\
\hline \multicolumn{5}{|l|}{ Pre-operative } \\
\hline Body temperature, ${ }^{\circ} \mathrm{C}$ & $36.21 \pm 0.39$ & $36.22 \pm 0.37$ & $36.2 \pm 0.46$ & 0.738 \\
\hline Heart rate $>100$ beats per minute, $n(\%)$ & $2(1.9)$ & $1(1.3)$ & $1(4.3)$ & 0.398 \\
\hline Time from admission to surgery, minutes & $214.54 \pm 109.37$ & $209.15 \pm 101.90$ & $233.30 \pm 133.06$ & 0.978 \\
\hline Hemoglobin, g/dL & $12.56 \pm 1.79$ & $12.65 \pm 1.84$ & $12.52 \pm 1.62$ & 0.350 \\
\hline White blood cell count, $10^{9} / \mathrm{L}$ & $7.13 \pm 2.35$ & $7.00 \pm 2.01$ & $7.60 \pm 3.29$ & 0.994 \\
\hline Sodium, mmol/L & $139.14 \pm 3.58$ & $139.25 \pm 3.61$ & $138.74 \pm 3.55$ & 0.478 \\
\hline Potassium, mmol/L & $4.19 \pm 0.51$ & $4.22 \pm 0.52$ & $4.12 \pm 0.47$ & 0.637 \\
\hline Creatinine, $\mathrm{mg} / \mathrm{dL}$ & $1.27 \pm 0.79$ & $1.22 \pm 0.31$ & $1.41 \pm 1.59$ & 0.068 \\
\hline Pain VAS score & $5.07 \pm 1.79$ & $4.98 \pm 1.77$ & $5.39 \pm 1.85$ & 0.289 \\
\hline \multicolumn{5}{|l|}{ Intra-operative } \\
\hline Type of surgery, N (\%) & & & & 0.134 \\
\hline Spine $e^{\pi}$ & $21(23.9)$ & $22(27.5)$ & $7(30.4)$ & \\
\hline $\mathrm{Hip}^{\dagger \dagger}$ & $36(40.9)$ & $25(31.1)$ & $10(43.5)$ & \\
\hline Knee ${ }^{\ddagger \ddagger}$ & $31(35.2)$ & $33(41.3)$ & $6(26.1)$ & \\
\hline General anesthesia & $46(44.7)$ & $37(46.3)$ & $9(39.1)$ & 0.637 \\
\hline Received blood transfusion during operation, $\mathrm{N}(\%)$ & $26(25.2)$ & $18(22.5)$ & $15(65.2)$ & 0.278 \\
\hline Lowest mean arterial pressure, $\mathrm{mm} \mathrm{Hg}$ & $71.54 \pm 10.97$ & $71.81 \pm 11.25$ & $70.60 \pm 10.15$ & 0.617 \\
\hline PCA, N (\%) & $20(19.4)$ & $17(21.3)$ & $3(13)$ & 0.552 \\
\hline
\end{tabular}

Data are presented as mean \pm SD or categorical variables in proportions number of patients (percentage). ${ }^{*} \geq 4$ prescriptions, ${ }^{\dagger}$ range 0 (completely dependent) to 100 (completely independent), ${ }^{\star}$ range 8 (completely dependent) to 0 (completely independent), \$range 0 -15, a cut-off vale of $\geq 5$ to indicate clinically important depressive symptoms, "range 1 (less painful sensation) to 10 (very painful sensation), " gery: includes spinal decompression only and spinal surgery with instrumented fusion, ${ }^{\text {th}}$ hip surgery: includes total hip replacement, bipolar hemiarthroplasty, revision hip surgery, and open reduction and internal fixation (ORIF), ${ }^{\ddagger \neq}$ knee surgery: includes total knee replacement and other elective knee surgery. BMI: Body Mass Index, CCI: Charlson Comorbidity Index, GDS-15: 15-item Chinese Geriatric Depression Scale, IADL: Instrumental Activity of Daily Living, MMSE: Mini-Mental State Examination, MNA-SF: short form Mini Nutritional Assessment, VAS: Visual Analogue Scale, POD: postoperative delirium 
Table 2. Group comparisons of baseline IGF-1 serum levels between subjects with POD and those without POD

\begin{tabular}{cccc}
\hline Variables & $\begin{array}{c}\text { All enrolled patients } \\
(\mathrm{N}=103)\end{array}$ & $\begin{array}{c}\text { Non-delirium patients } \\
(\mathrm{N}=80)\end{array}$ & $\begin{array}{c}\text { Delirium patients } \\
(\mathrm{N}=23)\end{array}$ \\
\hline Preoperative serum IGF-1 level $(\mathrm{ng} / \mathrm{mL})$ & $96.35 \pm 47.31$ & $96.14 \pm 47.58$ & $97.05 \pm 47.41$
\end{tabular}

Data are presented as mean \pm SD. IGF-1: insulin-like growth factor 1, POD: Postoperative delirium

\section{Statistical analyses}

Data were analyzed using SPSS v18. Baseline demographics and clinical characteristics are presented as median \pm standard deviation. Continuous and categorical variables are presented as an interquartile range and proportions, respectively. MannWhitney $\mathrm{U}$ and $\mathrm{t}$-tests were used to examine the difference in characteristics between patients who did and did not develop POD. These variables were chosen because of their clinically important significance for POD. ${ }^{1,2,46}$ Spearman's rank correlation coefficients were calculated to evaluate the relationship between baseline characteristics and serum IGF-1 levels. We performed a logistic regression to identify the association between serum IGF-1 levels and delirium status after adjustment of possible confounding factors. All results were twotailed and $\mathrm{p}<0.05$ was considered statistically significant.

\section{RESULTS}

Twenty-three out of 103 patients assessed on admission developed POD, as determined by DSM-IV TR and CAM criteria. $^{24}$ The patients were categorized into the POD $(n=23)$ and non-POD group $(n=80)$. Demographic and baseline characteristics of the patients are shown in Table 1. The POD group showed higher and lower CCI and MMSE scores, respectively, compared with the non-POD group. More emergency operations were performed in the POD group than the non-POD group (30.4\% vs. $10 \%$ ). No association was found between preoperative serum IGF-1 levels and POD in a univariate analysis (Table 2). In addition, serum IGF-1 levels were significantly correlated with MMSE scores and age (MMSE: $r=$ 0.230, $\mathrm{p}<0.05$; Age: $\mathrm{r}=-0.419, \mathrm{p}<0.001$ ), in accordance with previous findings. ${ }^{15,17}$ Studies have been found cognitive status and age were well documented risk factors to develop POD. ${ }^{1,6}$ Thus, a logistic regression analysis adjusting for MMSE and age was conducted to exclude these confounding factors. After adjustment, IGF-1 concentrations were not significantly associated with POD.

\section{DISCUSSION}

Serum biomarkers for delirium could be of three types: 1) risk markers that can be identified prior to disease onset to identify subjects at risk of developing delirium; 2) disease markers that occur with disease onset; and 3) markers that correlate with disease severity or consequence. ${ }^{28}$ Previous studies have shown an association between lower levels of IGF-1 and delirium; however, these studies do not consider the effect of acute illness in this relationship. For example, Egberts et al. ${ }^{10}$ found that 21 out of 23 patients who were diagnosed with delirium at the time of enrollment reported lower mean levels of IGF-1 compared with patients without delirium. Another study has reported that 42 out of 164 (25.6\%) patients were diagnosed with delirium at the time of blood sampling and had lower serum IGF-1 levels when compared with nondelirious control patients. ${ }^{7}$ Cytokines are non-specific and their expression can be altered by different physical conditions. Changes in cytokine levels arise after various insults, such as delirium; ${ }^{28}$ therefore, IGF-1 could serve as a suitable disease biomarker. ${ }^{7,810}$ However, it is unknown whether IGF1 is a risk marker because few studies that have been conducted assessing this and results show inconsistency. ${ }^{9,11-13}$

Abnormal IGF-1 levels have been implicated in several neurological and psychiatric disorders, such as Alzheimer's disease (AD), schizophrenia, and ischemic stroke. ${ }^{29-31}$ Serum IGF1 induces the clearance of amyloid beta, a pathological hallmark of $\mathrm{AD}$, from the brain via modulation of the expression of the carrier proteins transthyretin and albumin. A decline in circulating IGF-1 level reduces IGF-1 uptake in the brain and enhances AD development. ${ }^{29}$ Venkatasubramanian and colleagues assessed serum IGF-1 levels in 44 antipsychotic-naïve patients with schizophrenia and found that their levels were lower than those of control cases. ${ }^{30}$ In line with this, Bondanelli and colleagues suggested that patients who have ischemic stroke with higher levels of IGF-1 have better outcomes. ${ }^{31}$ These observations suggest that IGF-1 is neuroprotective. Furthermore, several studies have found that acute stress induces a reduction in serum IGF-1. ${ }^{32}$ The association between lower serum IGF-1 levels and the prevalence of delirium, including delirium at the time of admission, suggests, in part, that an alteration in IGF-1 levels is a consequence, rather than a cause, of delirium.

The present study could not replicate some of the previous findings of reduced serum IGF-1 in delirious patients., ${ }^{7,10,13}$ Differences in the methodological design and study population might explain this. We excluded patients who presented with delirium at the time of admission to reduce the effect of acute illness. In addition, the mean CCI score of patients in the present study was 1 and $74.6 \%$ of patients were undergoing 
elective operations. This suggests a relatively healthy population in this study sample. As such, we avoided the influence of confounding factors, such as infection, physical insult from an operation, or other comorbid conditions, on baseline serum IGF-1 levels. Additionally, the risk of delirium among patients who are preparing for an operation might differs from those of acutely ill inpatients who have higher comorbidities and poor physical status. ${ }^{33}$ Two studies conducted in orthopedic wards have shown no association between premorbid IGF-1 levels and incidence of delirium. ${ }^{9,11}$ Conversely, results are conflicting among studies on inpatients with acute illness. ${ }^{7,8,10,12}$ It is difficult to control for confounding factors in these patients because of the multifactorial nature of insults that can lead to delirium and relatively small sample size in most studies. Adamis and colleagues did not adjust for cognitive function and disease severity in their study, which may have impacted their results. ${ }^{7}$ Cognitive impairment is a significant risk factor for developing delirium in elderly hospitalized patients; therefore, not adjusting for this may lead to different conclusions. ${ }^{33}$ The use of an homogeneous population with less comorbidities, such as patients undergoing orthopedic surgery, may help to isolate the influence of biomarkers on postoperative delirium from patient- or illness-associated factors. ${ }^{5}$

Our findings were in agreement with some previous studies; ${ }^{9,11,12}$ however, we do find differences in our data. Lemstra and colleagues have reported that preoperative IGF-1 serum levels do not predict the development of POD in their study of elderly patients undergoing hip surgery (mean age, 80 years). However, gender is a main risk factor for POD and was not controlled for in the study. ${ }^{11}$ Cerejeira and colleagues enrolled younger surgical patients (mean age, 73 years) undergoing elective hip arthroplasty and found no association between IGF-1 levels andincidence of postoperative delirium. ${ }^{9}$ The innate immunity of the brain is more reactive in older people; ageing populations show exacerbated neuroinflammation when exposed to stress or comorbid conditions, which potentially leads to neurobehavioral changes and delirium. ${ }^{34}$ In the present study, we confirmed that preoperative IGF-1 levels were not associated with POD in an older population with same sex distribution.

Premorbid serum IGF-1 levels were not predictive of subsequent POD; however, dysfunctions in the growth hormone (GH)/IGF-1 axis are vital pathophysiological mechanisms of POD. Cerejeira and colleagues enrolled 101 non-demented patients aged 60 years and above who were undergoing elective hip arthroplasty and measured pre- and postoperative plasma IGF-1 levels. Preoperative IGF-1 levels were not different between patients with and without POD, similar to our findings; however, a greater elevation in IGF-1 relative to baseline was found in patients who developed POD. This impli- cates a dysregulation in the GH/IGF-1 axis; a decreased suppression of GH/IGF-1 signaling may be associated with POD. ${ }^{9}$ As we only measured preoperative serum IGF-1 in the present study, we were unable to measure any changes in the GH/ IGF-1 axis.

This study replicates the previous findings from western countries and confirmed no association between POD and serum IGF-1 levels in an Asian population. In accordance with Lemstra and colleagues, we did not find an association between preoperative IGF-1 levels and POD in our elderly population. ${ }^{11}$ However, this study had several limitations.

Firstly, our study is to apply the simplified model of markers of inflammation and stress system of predicting POD. Regard of this, considering complex interaction of IGF-1 with various cytokines such as IFN- $\gamma$, IL- 6 , TNF- $\alpha$, and IL- $1 \beta$ are needed. However, we did not measure these cytokines in our study and that may influence the pathophysiological effect of IGF-1 on delirium. Secondly, increases in IGF-1 levels during the course of acute illness have been reported. ${ }^{35}$ Therefore, sequential measurements of IGF-1 levels in serum before and at several time points after an operation could shed more light on this issue. Since we only measured IGF-1 at baseline, we were unable to examine potential associations between changes over time in IGF-1 concentrations and delirium. Future longitudinal studies including serial measurements of IGF-1 and other cytokines to better clarify the role of IGF-1 in the neurological effects of critical illness are warranted. Thirdly, the small sample size might lower the power of this study. Interpretation of our results should be cautious and cannot be generalized to the population as a whole. Fourth, serum concentrations of a biomarker that is derived from peripheral blood may not indicate the concentration of that biomarker in the brain. Future studies investigating the association between peripheral and central biomarker concentrations will be necessary. However, it may be difficult to identify a biomarker that significantly influences the process of delirium. Fifth, we cannot discount the contribution of dementia in these findings because cognitive impairment was strongly associated with the development of delirium. However, our regression analysis controlled for cognitive function and cognitive status could not explain the null association.

In conclusion, we did not find an association between preoperative IGF-1 serum levels and POD. This may not be a direct biomarker for predicting the incidence of POD among a relatively healthy elderly population. Further research with larger sample sizes is needed to clarify the relationship between biomarkers and neurological outcomes after surgical operations. 


\section{Acknowledgments}

We are grateful to our patients and their parents who voluntarily participated in our study and gave valuable information. This work was supported by grants VGHKS11-CT2-10 from Kaohsiung Veterans General Hospital, Taiwan.

\section{REFERENCES}

1. Amador LF, Goodwin JS. Postoperative delirium in the older patient. J Am Coll Surg 2005;200:767-773.

2. Lee HJ, Hwang DS, Wang SK, Chee IS, Baeg S, Kim JL. Early assessment of delirium in elderly patients after hip surgery. Psychiatry Investig 2011;8:340-347.

3. Liang CK, Chu CL, Chou MY, Lin YT, Lu T, Hsu CJ, et al. Interrelationship of postoperative delirium and cognitive impairment and their impact on the functional status in older patients undergoing orthopaedic surgery: a prospective cohort study. PLoS One 2014;9:e110339.

4. Yu KD, Lee TJ, Kim KI, Kim CH. Delirium in acute elderly care unit; prevalence, clinical characteristics, risk factors and prognostic significance. Psychiatry Investig 2005;2:80-82.

5. Chu CL, Liang CK, Lin YT, Chow PC, Pan CC, Chou MY, et al. Biomarkers of delirium: well evidenced or not? J Clin Gerontol Geriatr 2001;2:100-104.

6. Khan BA, Zawahiri M, Campbell NL, Boustani MA. Biomarkers for delirium--a review. J Am Geriatr Soc 2011;59(suppl 2):S256-S261.

7. Adamis D, Treloar A, Martin FC, Gregson N, Hamilton G, Macdonald AJ. APOE and cytokines as biological markers for recovery of prevalent delirium in elderly medical inpatients. Int J Geriatr Psychiatry 2007;22:688-694.

8. Adamis D, Lunn M, Martin FC, Treloar A, Gregson N, Hamilton G, et al. Cytokines and IGF-I in delirious and non-delirious acutely ill older medical inpatients. Age Ageing 2009;38:326-332.

9. Cerejeira J, Batista P, Nogueira V, Vaz-Serra A, Mukaetova-Ladinska EB. The stress response to surgery and postoperative delirium: evidence of hypothalamic-pituitary-adrenal axis hyperresponsiveness and decreased suppression of the GH/IGF-1 Axis. J Geriatr Psychiatry Neurol 2013;26:185-194.

10. Egberts A, Wijnbeld EH, Fekkes D, van der Ploeg MA, Ziere G, Hooijkass H, et al. Neopterin: a potential biomarker for delirium in elderly patients. Dement Geriatr Cogn Disord 2015;39:116-124.

11. Lemstra AW, Kalisvaart KJ, Vreeswijk R, van Gool WA, Eikelenboom P. Pre-operative inflammatory markers and the risk of postoperative delirium in elderly patients. Int J Geriatr Psychiatry 2008;23:943-948.

12. Morandi A, Gunther ML, Pandharipande PP, Jackson JC, Thompson JL, Shintani AK, et al. Insulin-like growth factor- 1 and delirium in critically ill mechanically ventilated patients: a preliminary investigation. Int Psychogeriatr 2011;23:1175-1181.

13. Wilson K, Broadhurst C, Diver M, Jackson M, Mottram P. Plasma insulin growth factor-1 and incident delirium in older people. Int J Geriatr Psychiatry 2005;20:154-159.

14. Adamis D, Meagher D. Insulin-like growth factor I and the pathogenesis of delirium: a review of current evidence. J Aging Res 2011;2011:951403.

15. Jones JI, Clemmons DR. Insulin-like growth factors and their binding proteins: biological actions. Endocr Rev1995;16:3-34.

16. Liu JP, Baker J, Perkins AS, Robertson EJ, Efstratiadis A. Mice carrying null mutations of the genes encoding insulin-like growth factor I (Igf1) and type 1 IGF receptor (Igf1r). Cell 1993;75:59-72.
17. Werner $\mathrm{H}$, LeRoith $\mathrm{D}$. Insulin and insulin-like growth factor receptors in the brain: physiological and pathological aspects. Eur Neuropsychopharmacol 2014;24:1947-1953.

18. Gluckman PD, Guan J, Williams C, Scheepens A, Zhang R, Bennet L, et al. Asphyxial brain injury--the role of the IGF system. Mol Cell Endocrinol 1998;140:95-99.

19. Hurlock EC 4th. Interferons: potential roles in affect. Med Hypotheses 2001;56:558-566.

20. Hwa V, Little B, Kofoed EM, Rosenfeld RG. Transcriptional regulation of insulin-like growth factor-I by interferon-gamma requires STAT-5b. J Biol Chem 2004;279:2728-2736.

21. Mahoney FI, Barthel DW. Functional evaluation: the Barthel Index. Md State Med J 1965;14:61-65.

22. D’Ath P, Katona P, Mullan E, Evans S, Katona C. Screening, detection and management of depression in elderly primary care attenders. I: The acceptability and performance of the 15 item Geriatric Depression Scale (GDS15) and the development of short versions. Fam Pract 1994;11: 260-266.

23. Magruder-Habib K, Stevens HA, Alling WC. Relative performance of the MAST, VAST, and CAGE versus DSM-III-R criteria for alcohol dependence. J Clin Epidemiol 1993;46:435-441.

24. Folstein MF, Folstein SE, McHugh PR. "Mini-mental state". A practical method for grading the cognitive state of patients for the clinician. J Psychiatr Res 1975;12:189-198.

25. Charlson ME, Pompei P, Ales KL, MacKenzie CR. A new method of classifying prognostic comorbidity in longitudinal studies: development and validation. J Chronic Dis 1987;40:373-383.

26. Swan IR, Browning GG. The whispered voice as a screening test for hearing impairment. J R Coll Gen Pract 1985;35:197.

27. Inouye SK, van Dyck CH, Alessi CA, Balkin S, Siegal AP, Horwitz RI. Clarifying confusion: the confusion assessment method. A new method for detection of delirium. Ann Intern Med 1990;113:941-948.

28. Marcantonio ER, Rudolph JL, Culley D, Crosby G, Alsop D, Inouye SK. Serum biomarkers for delirium. J Gerontol A Biol Sci Med Sci 2006; 61:1281-1286.

29. Carro E, Trejo JL, Gerber A, Loetscher H, Torrado J, Metzger F, et al. Therapeutic actions of insulin-like growth factor I on APP/PS2 mice with severe brain amyloidosis. Neurobiol Aging 2006;27:1250-1257.

30. Venkatasubramanian G, Chittiprol S, Neelakantachar N, Naveen MN, Thirthall J, Gangadhar BN, et al. Insulin and insulin-like growth factor-1 abnormalities in antipsychotic-naive schizophrenia. Am J Psychiatry 2007;164:1557-1560.

31. Bondanelli M, Ambrosio MR, Onofri A, Bergonzoni A, Lavezzi S, Zatelli MC, et al. Predictive value of circulating insulin-like growth factor I levels in ischemic stroke outcome. J Clin Endocrinol Metab 2006; 91:3928-3934.

32. Mesotten D, Van den Berghe G. Changes within the growth hormone/ insulin-like growth factor I/IGF binding protein axis during critical illness. Endocrinol Metab Clin North Am 2006;35:793-805.

33. Elie M, Cole MG, Primeau FJ, Bellavance F. Delirium risk factors in elderly hospitalized patients. J Gen Intern Med 1998;13:204-212.

34. Godbout JP, Johnson RW. Age and neuroinflammation: a lifetime of psychoneuroimmune consequences. Neurol Clin 2006;24:521-538.

35. Baxter RC, Hawker FH, To C, Stewart PM, Holman SR. Thirty-day monitoring of insulin-like growth factors and their binding proteins in intensive care unit patients. Growth Horm IGF Res1998;8:455-463. 